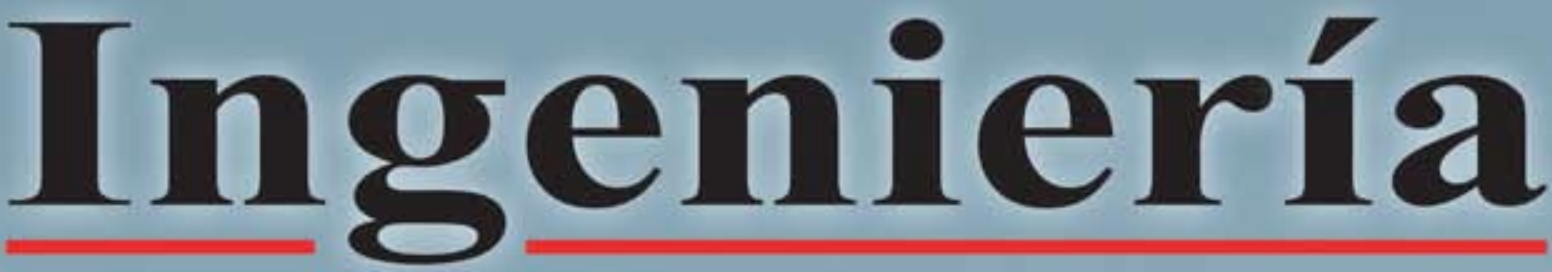

ENERO/DICIEMBRE 2004 - VOLUMEN 14 - N¹ y 2

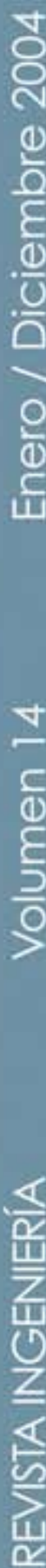
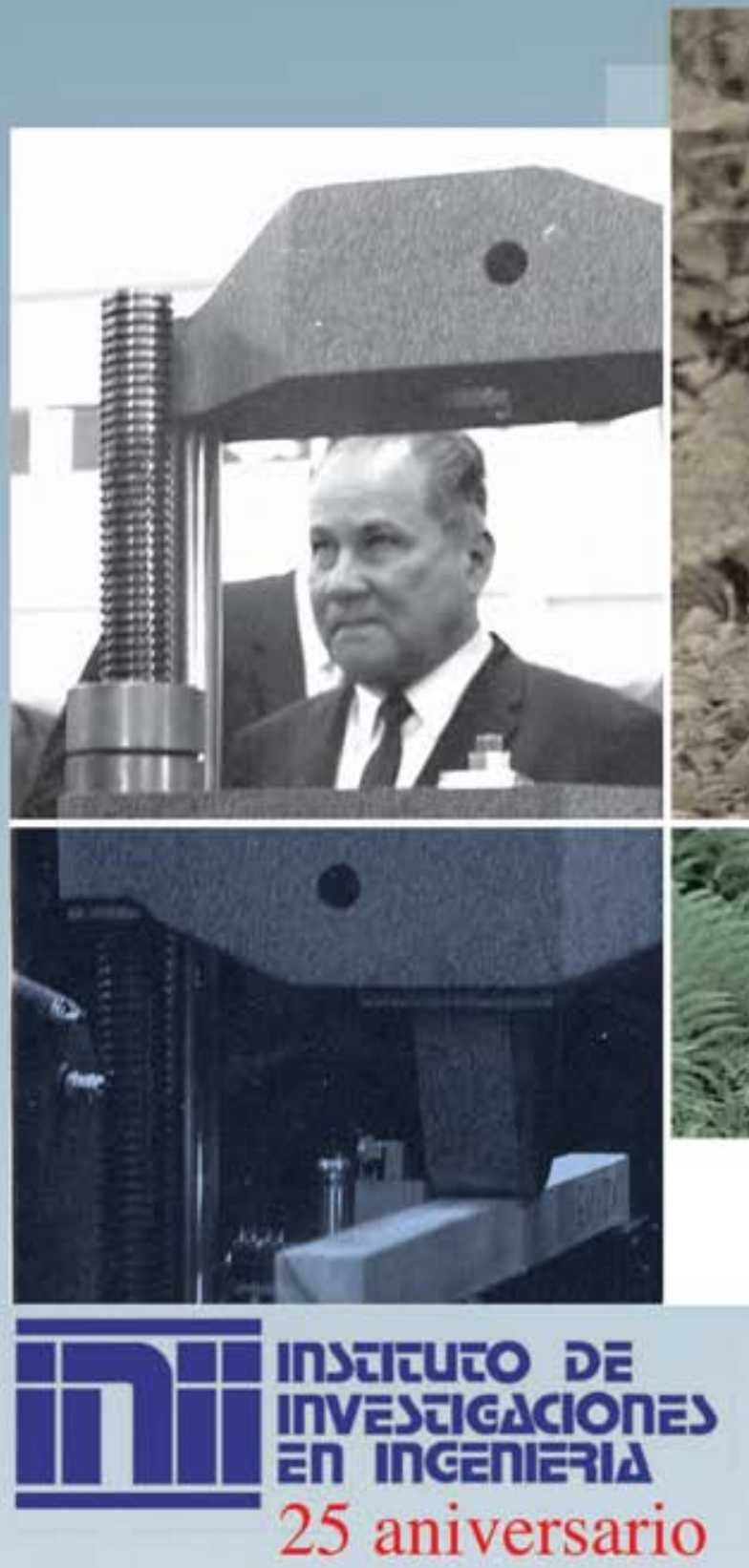

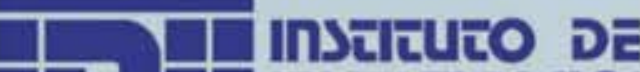
T W Investcscines हn Incenlei|d 25 aniversario

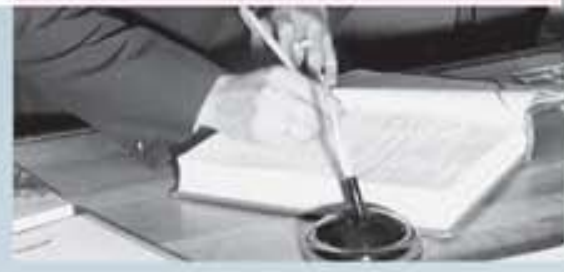




\title{
RECUPERACIÓN DE LA INFRAESTRUCTURA HOSPITALARIA EN EL SALVADOR, DESPUÉS DE LOS SISMOS DE ENERO Y FEBRERO DEL 2001
}

\author{
Miguel Francisco Cruz Azofeifa
}

\begin{abstract}
Resumen
Se presenta una descripción de los daños sufridos en el sistema hospitalario de El Salvador durante los sismos del 13 de enero y del 13 de febrero del 2001.

Se enfatiza que el daño se concentró básicamente en los aspectos no estructurales, lo cual se produjo por la alta flexibilidad de las estructuras. Este daño y la falta de conocimiento de la verdadera resistencia de las estructuras, dejaron fuera de servicio a los hospitales, Provocando incertidumbre hacia la respuesta estructural ante próximos sismos.

El Hospital Santa Teresa es una muestra de esta situación, pues sufrió daño no estructural leve durante el primer sismo y daños no estructural y estructural severo durante el segundo sismo. Además, se presenta un análisis de las deformaciones laterales, del Hospital Santa Teresa durante los dos sismos, a efectos de establecer el límite de desplazamiento donde los daños no estructurales empiezan a ser importantes, asi como la estrategia de recuperación hospitalaria propuesta al gobierno salvadoreño.
\end{abstract}

Se valoran el tiempo y los costos de dicha recuperación.

Palabras clave: hospitales, daño sísmico, daño no estructural.

\begin{abstract}
A damage description suffered by the El Salvador hospitals during the earthquakes of January and February 2001 is presented in this work.

It is emphasized that the damage was basically non-structural damage, which was caused by the high flexibility of the structures. This damage and the lack of knowledge about the real strength of the structures putted the hospitals out of service until confidence could be achieved about the structural behavior during future earthquakes.
\end{abstract}

Sta. Teresa Hospital showed this situation; it suffered light non - structural damage during the first quake and suffered extensive non - structural and structural damage during the second quake. A lateral displacement analysis is performed to Sta. Teresa Hospital due to the earthquakes, in order to establish a lateral displacement limit to control the non -structural damage.

It is also presented a hospital recovery strategy proposed to the El Salvador Government. The time and cost of this recovery are estimated.

Keywords: hospitals, earthquake damage, non-structural damage.

Recibido 06-X-04 • Aceptado 18-XI-04

\section{INTRODUCCIÓN}

El 13 de enero del 2001, la República de El Salvador fue sacudida por un sismo de magnitud 7,6 (momento) que causó intensidades máximas en el territorio nacional de 8 en la escala de Mercalli Modificada (MM). Este sismo causó daño moderado en la red hospitalaria del primer nivel de atención y gran daño en la red hospitalaria del segundo nivel.

El 13 de febrero del mismo año ocurrió un segundo sismo de magnitud 6,5 (momento) que causó intensidades máximas de VIII (MM) en 
la zona epicentral. Este sismo fue de carácter local y continuó dañando la red hospitalaria del segundo nivel, sin causar más daño en la red primaria.

Los dos sismos, en conjunto, causaron una pérdida total en todo el país de $\$ 1600$ millones. El sector vivienda de ingresos medios y bajos resultó muy afectado, con 75000 viviendas destruidas y 27800 viviendas dañadas. La red vial sufrió daños a lo largo de $400 \mathrm{~km}$ y su recuperación mantuvo una crisis en el sector transporte. Los sectores de educación y de salud fueron los más impactados a nivel gubernamental.

\section{DAÑO HOSPITALARIO CAUSADO POR LOS SISMOS DEL 13 DE ENERO Y DEL 13 DE FEBRERO DEL 2001}

El daño en el sistema hospitalario fue muy variado, dependiendo del tipo hospital y del tipo de edificación. El Cuadro 1 muestra el número de camas y el porcentaje dañado de estas los hospitales del primer y segundo nivel, pertenecientes al Ministerio de Salud. Puede notarse que en los hospitales del tercer nivel no hubo pérdida de camas debido al daño moderado presentado en ellos.

El impacto más grande se dio en los hospitales del segundo nivel en los que se perdió un $32,5 \%$ de las camas, debido al extenso daño no estructural y algún daño estructural en cuatro importantes hospitales regionales. Los hospitales del primer nivel no tuvieron pérdidas de camas, sin embargo, las áreas de quirófanos debieron salir de operación dado el daño no estructural.

El Cuadro 1 indica que hubo 32 camas perdidas en el Hospital de Niños Benjamín Bloom; sin embargo, este hospital no sufrió ningún tipo de daño. La situación de pérdida de camas en este hospital se debió a que el personal del octavo piso decidió abandonar el servicio dadas las fisuras que presentaban las paredes de relleno. El edificio consta de 11 pisos y fue reforzado después del sismo de 1986; el octavo piso, fue el punto donde se dio el cambio de rigidez del reforzamiento, el agrietamiento fue mínimo, por lo que se consideró que la actitud del personal fue injustificada.

Cuadro 1. Camas en los Hospitales del Ministerio de Salud y Asistencia Social.

$\begin{array}{lccc}\text { Hospital } & \text { Camas Totales } & \text { Camas Perdidas } & \text { Camas Perdidas } \\ \text { De primer nivel } & 1 & & (\%) \\ \text { Rosales } & 137 & 32 & 2,8 \\ \text { Benjamín Bloom } & 547 & 0 & 0 \\ \text { Dr. Argüello Escolán, Maternidad308 } & 282 & 32 & 11 \\ \text { De segundo nivel } & 0 & 0 & 32,5 \\ \text { San Juan de Dios, Santa Ana } & 2716 & 883 & 0 \\ \text { Dr. Luis E. Vázquez, Chalatenango } & 469 & 0 & 0 \\ \text { San Rafael } & 100 & 0 & 59 \\ \text { Cojutepeque } & 228 & 135 & 0 \\ \text { Santa Teresa, Zacatecoluca } & 78 & 0 & 100 \\ \text { Santa Gertrudis, San Vicente } & 165 & 165 & 0 \\ \text { San Juan de Dios, San Miguel } & 139 & 0 & 100 \\ \text { Dr. José Molina Martínez, Psiquiátrico } & 420 & 420 & 0 \\ \text { San Pedro, Usulután } & 432 & 0 & 100 \\ \text { Dr. José Saldaña, Neumológico } & 138 & 138 & 9 \\ \text { Dr. Juan José Fernández, Zacamil } & 292 & 25 & 0\end{array}$


A continuación se expone el tipo de daño que presentaron cuatro hospitales regionales del segundo nivel y que, a criterio del autor, son los que mantienen al sistema hospitalario en condiciones de inoperabilidad indefinida y que requiere de una intervención a fondo, a fin de restablecer el servicio habitual.

\section{Hospital San Rafael (Santa Tecla)}

Este hospital está compuesto por una serie de construcciones de diferente tipo y antigüedad. Existían en el hospital, antes del sismo, múltiples edificios de adobe de un piso, declarados patrimonio nacional, que resultaron severamente dañados con el sismo del 13 de enero, y lo cual requirió su demolición. Esto hizo que se perdieran áreas de almacenamiento y bodegas en el hospital. Existen también en el hospital algunos edificios de mampostería reforzada de uno y dos pisos que no sufrieron daño alguno.

La torre principal de hospitalización es un edificio de 6 pisos estructurado a base de marcos de concreto reforzado y con paredes de relleno de mampostería de barro sin refuerzo. El edificio tiene una forma $\mathrm{H}$ irregular de su planta de distribución, la cual fue dividida en cinco cuerpos independientes.
Los daños en las paredes de relleno de fachada e interiores son extensivos, lo mismo que en las juntas de dilatación. El daño en pisos y cielos es extensivo y no puede lograrse una recuperación rápida del nosocomio. El daño no estructural tan extensivo mencionado, se debe a la gran flexibilidad del sistema estructural que a la postre terminó apoyándose en las paredes (no estructurales) causando agrietamiento en ellos.

\section{Hospital San Pedro (Uzulután)}

Este edificio está compuesto por siete edificios de concreto reforzado. En la Figura 1, se muestra esta distribución.

Este hospital resultó dañado por el sismo del 13 de enero y sin daño adicional durante el sismo del 13 de febrero. El edificio central, de siete pisos, está compuesto por un sistema de muros de corte y alberga a las escaleras y a los ascensores. Los edificios administrativos son edificios de un piso. Estos edificios, al igual que el edificio central, no presentaron daño alguno. Los edificios de hospitalización y quirófanos, son edificios de seis pisos estructurados a base de marcos de concreto reforzado, muy flexibles y con paredes de relleno de mampostería de

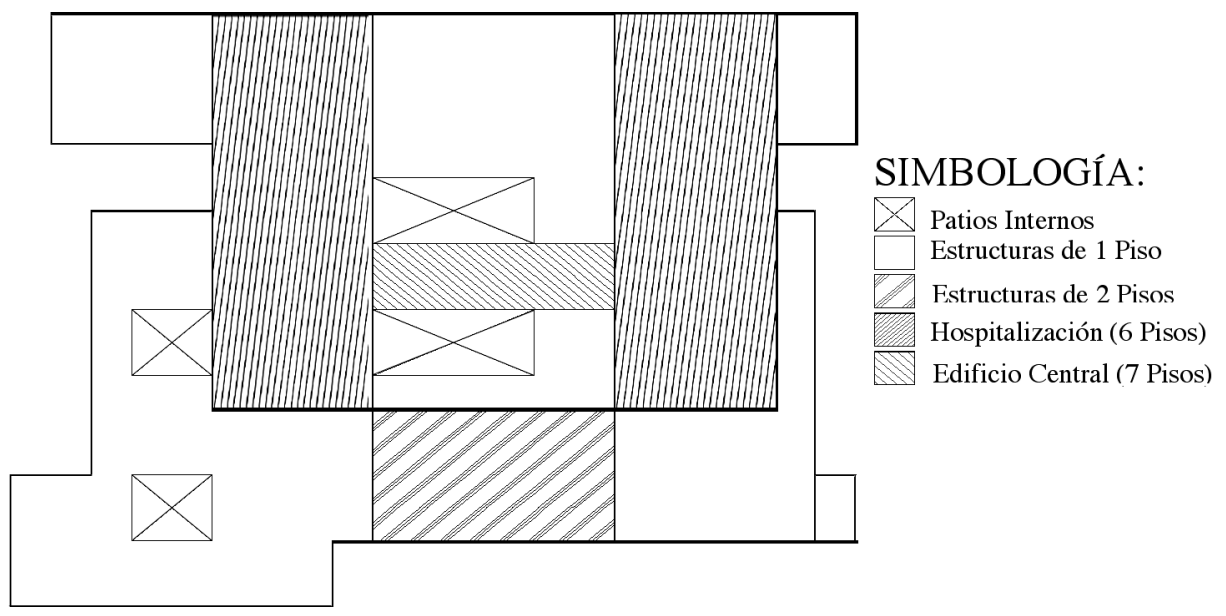

Figura 1. Distribución en planta del Hospital San Pedro. 
concreto, reforzada y desligada en la estructura. La separación entre paredes y estructura indicada en los planos es de $2,5 \mathrm{~cm}$; sin embargo, en la obra solo se dejó entre $2 \mathrm{~cm}$ y $1 \mathrm{~cm}$.

En muchos puntos se dejó la separación en la estructura, pero no en los repellos, lo que causó el agrietamiento de los mismos de manera extensiva, así como agrietamientos de la pared. Algunas paredes de relleno no alcanzan la altura total entre piso y cielo, sobre todo en las fachadas para formar las ventanas, lo que provocó que se presentara el fenómeno de columna corta en el primer piso. Esta condición de falla estructural, adicionada al daño extensivo en pisos, paredes y cielos compromete el servicio hospitalario y deja al hospital en una condición de recuperación lenta.

La alta flexibilidad de la estructura en los edificios de hospitalización, provocó el daño no estructural y sacó al hospital de servicio de manera indefinida, hasta tanto no se reparen los daños, labor que no podrá lograrse a corto plazo.

\section{Hospital Santa Teresa (Zacatecoluca)}

El hospital de Zacatecoluca es un hospital gemelo del de Usulután, construido con los mismos planos. Este hospital sufrió un daño muy leve en los componentes no estructurales durante el sismo del 13 de enero; sin embargo, el sismo del 13 de febrero vino a causar extensivo daño no estructural en los edificios de hospitalización y produjo daño en las columnas cortas de las fachadas. De esta manera, el daño general del hospital de Zacatecoluca durante el sismo del 13 de febrero resultó similar al daño presentado en el hospital de Usulután durante el sismo del 13 de enero.

El hospital de Zacatecoluca quedó inhabilitado después de este segundo sismo y, al igual que el de Usulután, su recuperación no se podrá dar a corto plazo. Más adelante se comentará la respuesta sísmica del hospital de Zacatecoluca durante cada sismo.

\section{Hospital San Juan de Dios (San Miguel)}

Este es el hospital regional más importante de la zona occidental de El Salvador, con 390 camas.

Está formado por un conjunto de cuatro estructuras independientes de seis pisos que albergan a la hospitalización y un núcleo central de siete pisos, que alberga las escaleras y los ascensores.

Cada parte está estructurada con pórticos de concreto reforzado y con paredes de relleno de mampostería de arcilla, desligadas de la estructura principal. La construcción de las paredes no siguió una práctica correcta, ya que algunas de ellas cuentan con repellos de hasta $5 \mathrm{~cm}$ de espesor.

Los daños que presentó el hospital después del sismo del 13 de enero, fueron extensivos en pisos, cielos y paredes de mampostería, en el núcleo central. El daño en este edificio, clave para la circulación vertical dentro del hospital, compromete el uso y el servicio del mismo. El núcleo central es tan flexible, que su estructura se apoyó en las paredes de relleno y, por lo tanto, muchas paredes tienen agrietamiento excesivo y las de la planta baja, muestran un desplome que las hace muy peligrosas para los usuarios. Los edificios de hospitalización no sufrieron daño de ningún tipo, salvo pequeñas grietas en las paredes de relleno que no comprometen el servicio.

En general, el daño causado por los sismos mencionados al sistema hospitalario salvadoreño perteneciente al Ministerio de Salud, fue básicamente no estructural y el daño estructural observado en los hospitales de Usulután y Zacatecoluca, se debió a la interacción inapropiada de los elementos no estructurales con la estructura.

Una situación similar se vivió en los hospitales pertenecientes a la Seguridad Social, donde tres hospitales en San Salvador y el de Sonsonate, debieron salir de servicio por daños excesivos, en lo no estructural. 
Estos eventos sísmicos vinieron a demostrar una vez más que las estructuras flexibles permiten, en la mayoría de los casos, sobrevivir el sismo sin daño estructural; sin embargo, el daño no estructural de elementos frágiles que interactúan con la estructura se hace extensivo y deja a los nosocomios fuera de servicio, agravando la atención de la emergencia en los días posteriores al sismo.

\section{ANÁLISIS DE LOS DESPLAZAMIENTOS DEL HOSPITAL DE ZACATECOLUCA}

Como ya se mencionó, este hospital sufrió daño únicamente durante el sismo del 13 de febrero y el daño se ubicó, principalmente, en los edificios de hospitalización. Los edificios de hospitalización son edificios de seis pisos estructurados a base de marcos de concreto flexibles. En la Figura 2 se muestra la forma de un edificio de hospitalización.

Se observa que existen trece marcos de tres columnas en la dirección transversal y tres marcos de trece columnas en la dirección longitudinal. El eje central longitudinal, contiene múltiples paredes de mampostería, separadas de la estructura $2 \mathrm{~cm}$ con materiales flexibles; sin embargo, el repello de las paredes se encuentra sobre las juntas y podría decirse que es el elemento de unión entre estructura y pared. En las fachadas, las paredes están hasta media altura a efectos de dejar espacio para las ventanas. La separación en estas paredes con la estructura es de $1 \mathrm{~cm}$. Las columnas tienen dimensiones de $70 \mathrm{~cm}$ por $35 \mathrm{~cm}$ en los dos primeros pisos y disminuyen su sección en cada piso, hasta llegar a $35 \mathrm{~cm}$ en el último piso. Los entrepisos son de losa colada en sitio, de $15 \mathrm{~cm}$ de espesor y con vigas de dimensiones de $30 \mathrm{~cm}$ por $70 \mathrm{~cm}$. El peso de cada piso alcanza los $1100 \mathrm{~kg} / \mathrm{m}^{2}$.

Para analizar los desplazamientos del edificio en la dirección longitudinal (dirección flexible) se hace uso del método estático elástico (Colegio Federado de Arquitectos e Ingenieros, 1986) y de los modelos estructurales de los ejes longitudinales mostrados en la Figura 3.

Este modelo, considera que, al inicio del sismo, la estructura de vigas y columnas soporta sola el sismo (modelo 1). Una vez cerrados los espacios entre paredes y estructuras, las paredes empiezan a tomar cargas, a modificar los períodos de oscilación y a tratar de limitar las deformaciones (modelo 2). El daño secuencial de columnas cortas y de paredes, modifica nuevamente la respuesta estructural. Los tres estados de la estructura: vigas y columnas actuando solas, vigas y columnas

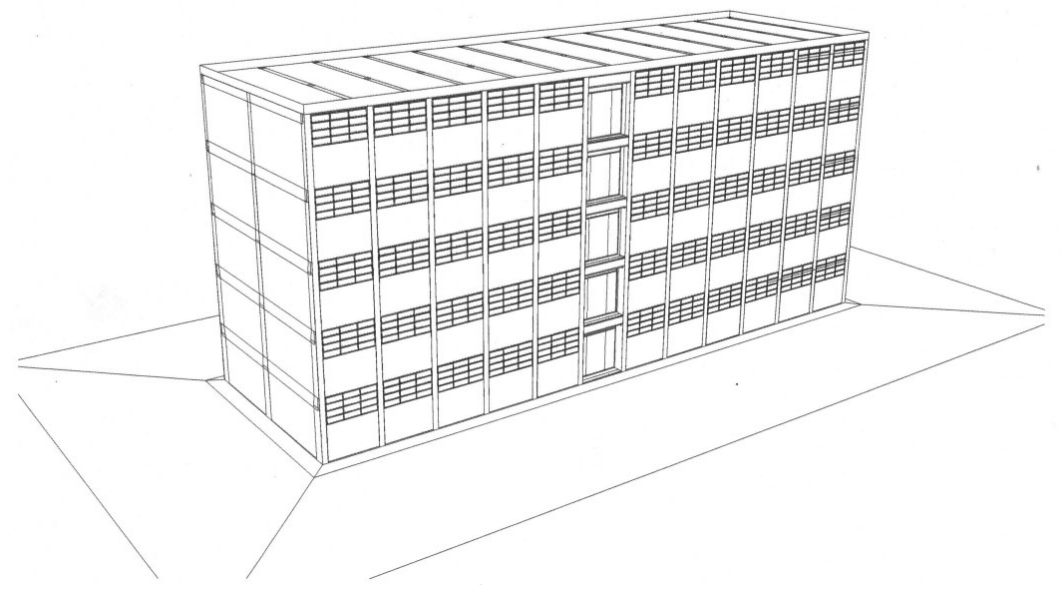

Figura 2. Elevación Hospital de Zacatecoluca 
interactuando con las paredes, y la falla de paredes y columnas cortas, se evaluarán con el análisis de desplazamiento siguiente.

Para el cálculo de las fuerzas, se utiliza el espectro unitario elástico mostrado en la Figura 4. Como el período original de la estructura corresponde únicamente al esqueleto de vigas y columnas, y el mismo es de $1,07 \mathrm{~s}$, se tiene un factor espectral dinámico de 1,28 , siendo 2,5 el valor máximo para períodos menores a $0,55 \mathrm{~s}$.

Con este factor espectral dinámico se calculan las fuerzas sísmicas sobre el esqueleto y con las fuerzas se obtendrán desplazamientos máximos del orden de $14 \mathrm{~cm}$ en el último piso para una fuerza total en la base de 900 ton, calculada con una aceleración máxima en la base de 0,40 g. Esta condición de carga-deformación se muestra en la Figura 5. El esqueleto no tiene libertad de moverse con todos los desplazamientos que le demanda el sismo.

Cuando el desplazamiento del último piso alcanza $8 \mathrm{~cm}$ (4 por mil de deformación relativa), se cierran los espacios entre la estructura y las paredes en el primero y en el segundo piso. A partir de este desplazamiento y en el supuesto de que todas las separaciones estén cerradas, el período de la estructura cambiaría a 0,71 y por consiguiente, las fuerzas sísmicas. El modelo con las separaciones cerradas, considera a las paredes como elementos diagonales según se describe en (Pauly y Priestley, sa).

En el nuevo modelo se verifican las condiciones de resistencia de las columnas cortas y de las paredes. Esta condición se muestra también en la Figura 5.

Como se puede observar, las fallas de las columnas cortas se alcanzan para deformaciones del último piso cercano a los $11 \mathrm{~cm}$ (6 por mil de deformación relativa) y la falla en compresión de las paredes se alcanza para deformaciones cercanas a los $13 \mathrm{~cm}$ (7 por mil). La falla en tracción de las paredes debió alcanzarse para deformaciones menores a $13 \mathrm{~cm}$.
Como este es un análisis elástico, las deformaciones aquí calculadas serán proporcionales a la aceleración máxima del movimiento sísmico. En la Figura 7 se muestra una escala de aceleración en el eje horizontal capaz de producir el desplazamiento del último piso, también graficado en el eje horizontal. Puede observarse que si la aceleración es menor que $0,24 \mathrm{~g}$, no logran cerrarse las aberturas entre paredes y estructura, es decir, la deformación relativa se mantiene inferior al 4 por mil.

El sismo del 13 de enero causó aceleraciones máximas en Zacatecoluca de $0,25 \mathrm{~g}$ en la dirección N-S y 0,20 en la dirección E-W (fuente Universidad Católica José Simón Cañas). Este sismo apenas si logró cerrar la separación entre estructura y paredes y de ahí el poco daño no estructural observado. Si la aceleración máxima del terreno fuese más grande, entonces podrían presentarse las fallas de las columnas y las fallas de las paredes.

La Figura 5 indica que las columnas y las paredes fallan para deformaciones entre $11 \mathrm{~cm}$ y $13 \mathrm{~cm}$, es decir, para deformaciones relativas entre un 6 mil y un 7 mil. Estas deformaciones serían causadas por registros con aceleraciones máximas entre 0,20 g y 0,42 g.

El sismo del 13 de febrero causó aceleraciones máximas en Zacatecoluca iguales a $0,40 \mathrm{~g}$ en la dirección N-S y $0,30 \mathrm{~g}$ en la dirección E-W (fuente: Universidad Católica JSC), razón por la cual las paredes y las columnas fallaron durante este sismo.

Este análisis de deformaciones y aceleraciones máximas del movimiento del terreno se correlaciona muy bien con el daño observado en el hospital. Puede decirse, que, mientras la deformación lateral relativa no superó el 4 por mil, el daño no estructural no se presentó. El daño estructural se alcanzó para deformaciones relativas del orden del 7 por mil, sin embargo esto se debe a la incorrecta interacción de la estructura con las paredes no estructurales. 


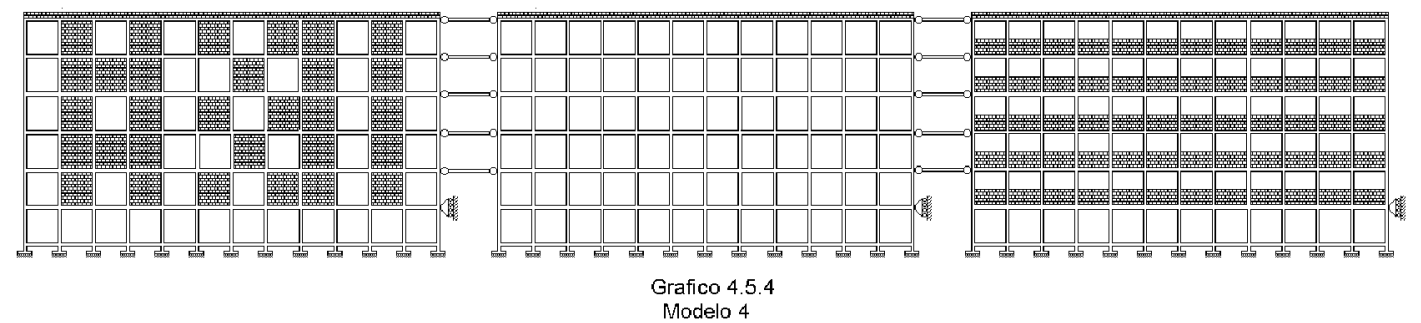

Figura 3. Modelo de ejes longitudinales

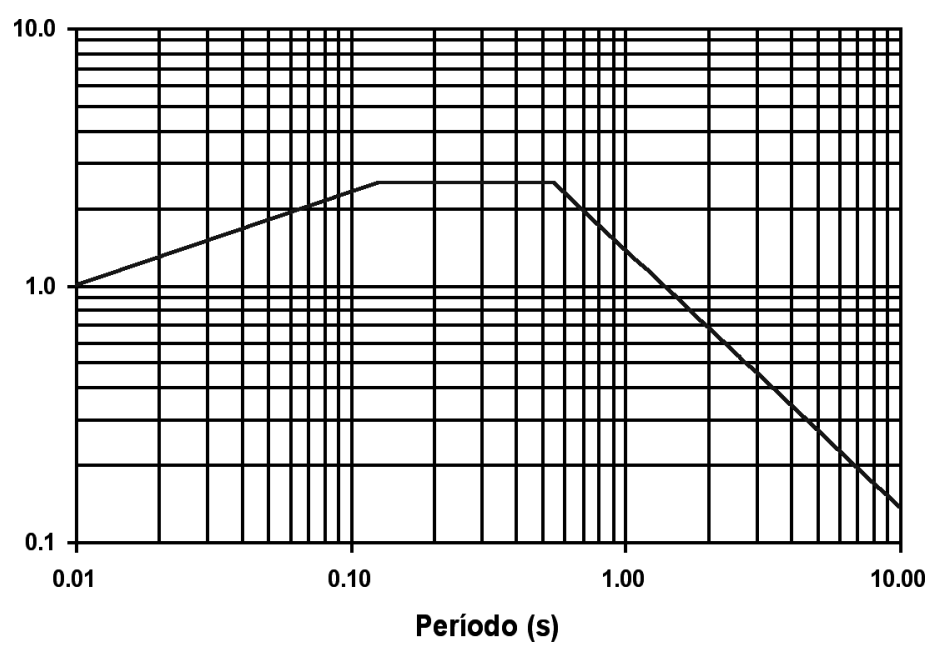

Figura 4. Espectro elástico unitario de aceleraciones

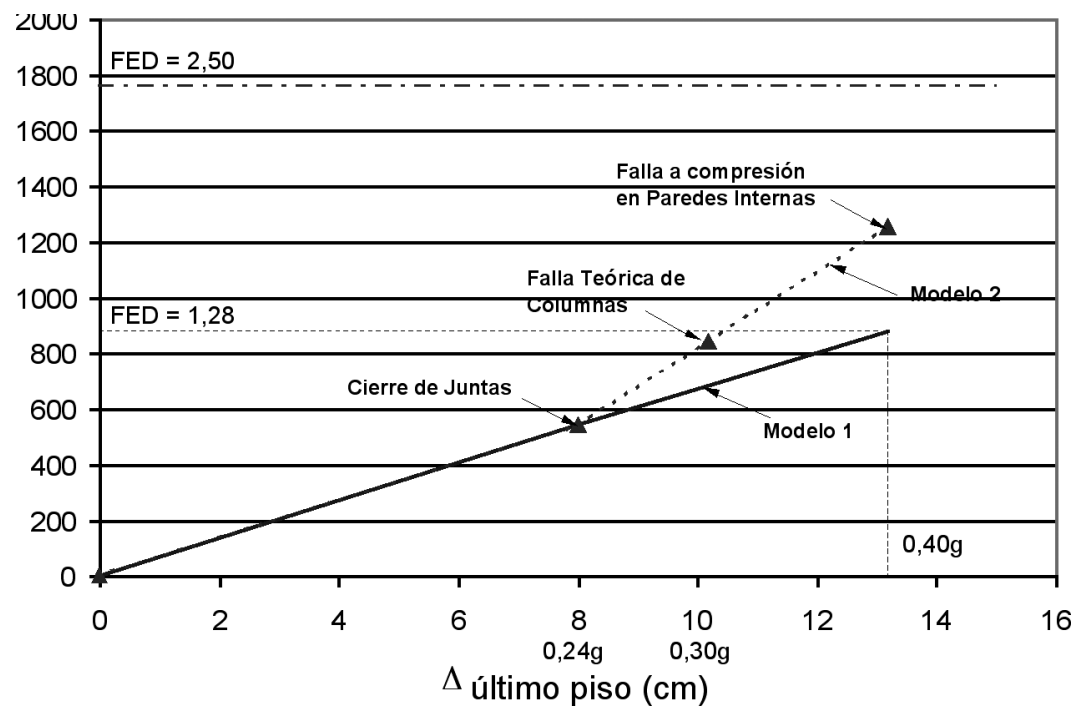

Figura 5. Cortante en base - desplazamiento del último piso 
Lo que sí es claro es que el límite del 25 por mil $(2,5 \%)$ admitido por las normas del (International Code Council, 2000) resultaría insuficiente para controlar el daño no estructural, en este tipo de estructura.

\section{PLAN DE RECUPERACIÓN DE LA RED HOSPITALARIA}

El nivel de daño sufrido por los hospitales de El Salvador, ha sido agravado por el estado de deterioro y obsolescencia de algunos nosocomios que obliga no solo a reparar los daños, sino a redefinir servicios y por consiguiente, a modificar la planta física.

En el Cuadro 2 se muestran los porcentajes de hospitales dañados en cada nivel y el tipo de intervención que requieren. Como puede observarse, un alto porcentaje requiere algún tipo de intervención y un alto grado muestra obsolescencia y vulnerabilidad.

Esta situación pone a la República de El Salvador ante la necesidad de asignar recursos al sector salud, a fin de recuperar y modernizar su red hospitalaria.

\section{Dificultad de la recuperación}

La recuperación hospitalaria no puede ser inmediata ya que debe realizarse una evaluación del estado de cada hospital, una definición de lo que debe lograrse y un diseño del refuerzo y modificación de la planta física. Este proceso de recuperación en los cuatro hospitales del segundo nivel comentados en el apartado 2, debe darse en condiciones de trabajo bastante restringidas, ya que será necesario recurrir a instalaciones provisionales.

La recuperación rápida en estos hospitales no puede darse debido a diversos factores que se comentan a continuación:

- Factores sociales y psicológicos: a pesar de que el daño es únicamente no estructural en la mayoría de los casos, la población salvadoreña no confía en la seguridad de sus edificios hospitalarios, y al menor signo de agrietamiento y la caída de objetos, la tendencia es de abandonar las instalaciones sin recurrir a los planes de evacuación existentes en los hospitales. Una vez desalojado el edificio, no hay argumento posible que los haga regresar al hospital. Esta actitud puede deberse a la historia sísmica de El Salvador, que ha sufrido innumerables pérdidas económicas y de vidas humanas debido al pobre comportamiento del adobe $\mathrm{y}$ las construcciones tradicionales. Aun el concreto reforzado se ha comportado pobremente en las últimas décadas.

- Factores financieros y administrativos: el reparar un hospital a corto plazo y decidir su reutilización, después de la observancia de daño generalizado, aún siendo no estructural, es un asunto de administración pública e implica la canalización de recursos y la asignación de un profesional responsable que determine el grado de seguridad del edificio.

Si estuviese dañado únicamente un hospital, este procedimiento podría darse como

Cuadro 2. Cuantificación de daños en hospitales (\%)

$\begin{array}{lccc} & \text { Primer nivel } & \text { Segundo nivel } & \text { Tercer nivel } \\ \text { Hospitales que pueden operar normalmente } & 33 & 40 & 100 \\ \text { Hospitales que requieren intervención } & 33 & 50 & 0 \\ \text { Hospitales que requieren intervención y reasignación de servicios } & 33 & 10 & 0 \\ \text { Hospitales con obsolescencia y alto grado de vulnerabilidad } & 66 & 90 & \text { indefinido }\end{array}$


máximo en una semana; sin embargo, ante el estado de emergencia del sistema hospitalario, este procedimiento no se pudo dar, aún con el apoyo de organismos internacionales, ya que el daño no estructural tan extensivo tiene su origen en una estructura flexible y vulnerable, por lo que habría que replantear la estructura para corregir el origen de los daños.

- Factores técnicos: como ya se mencionó, el daño no estructural tiene origen en la flexibilidad de la estructura y por lo tanto su reparación implica una reestructuración del sistema sismorresistente, lo que necesariamente demanda un diseño estructural. El diseño estructural requiere una licitación pública para escoger al diseñador y requiere también un estudio de diagnóstico. Esto no puede darse a corto plazo y la administración pública no puede omitir este diseño en aras de reparar apropiadamente un hospital.

En el caso de los cuatro hospitales mencionados, será necesario recurrir a un refuerzo estructural para corregir los problemas de comportamiento y por lo tanto, a un diseño del mismo.

\section{Plazo y costo de la recuperación}

Es claro que el proceso de recuperación de la red hospitalaria tomará tiempo y demandará recursos que habrá que asignar del presupuesto nacional. Mientras la recuperación se da, el Ministerio de Salud deberá realizar las siguientes acciones a fin de atender la demanda en los hospitales.

- Recuperación y puesta en funcionamiento de la máxima capacidad disponible. Los hospitales no se encuentran dañados en el $100 \%$. Habrá pisos, edificios y locales dentro de cada hospital que pueden utilizarse con o sin cambio de su uso habitual.

- Construcción de instalaciones temporarias: todos los hospitales cuentan con suficiente área útil para construir instalaciones temporarias, donde puedan brindarse algunos de los servicios de los pisos perdidos.

- Adquisición de locales para descomprimir la demanda: el alquiler o compra de locales aledaños al hospital puede darse para brindar algunos servicios como consulta externa, laboratorio, etc. y así crear espacio dentro de las áreas y edificios remanentes en el hospital.

- Realización de acuerdos con hospitales privados y/o de las fuerzas armadas: estos acuerdos podrían servir para cubrir parte de la demanda en algunas especialidades.

- Realización de acuerdos con países vecinos: estos acuerdos pueden darse a fin de cubrir cirugías en algunas especialidades.

La recuperación de la red requerirá, a su vez, una serie de pasos que deben darse en forma secuencial y que se pueden enumerar como sigue:

- Estudios de vulnerabilidad y diagnóstico de los hospitales existentes: es evidente que los hospitales que presentan daño son los primeros en recibir el estudio de diagnóstico del estado actual. Otros hospitales existentes que no presentan daño deben ser analizados mediante un estudio de vulnerabilidad. Es cierto que la intervención en los mismos es de menor prioridad que en los hospitales dañados, sin embargo, es necesario conocer su estado a efectos de proyectar su uso en el diseño de la nueva red.

- Estudio y determinación de la red hospitalaria: simultáneamente con los estudios de diagnóstico y vulnerabilidad de los hospitales, se debe iniciar un estudio que determine las necesidades de la red hospitalaria salvadoreña. Este estudio debe indicar, cuál servicio debe mantenerse en cada hospital, cual reducirse o ampliarse, e inclusive cuáles podrían desaparecer. El estudio debe culminar con una planificación que permita ordenar los servicios que ofrece 
la red de salud y que defina los perfiles de atención en cada hospital. Estos perfiles de atención deben ser definidos en función de las demandas de la población, y de los objetivos que el estado se proponga lograr en el sector salud. Con este panorama se podrá saber cuáles hospitales o parte de ellos pueden ser reforzados o ampliados.

- Proyecto de arquitectura, equipamiento, mantenimiento $y$ reforzamiento: los estudios de diagnóstico y de las necesidades y modificaciones de la red, permitirán definir el alcance del reforzamiento de cada hospital dañado y de los que resulten vulnerables, así como las modificaciones de su planta física. Los diseños se realizarán para reducir la vulnerabilidad y para que cumplan con las necesidades establecidas en el estudio de la red.
- Ejecución de los proyectos: una vez realizados los diseños de los proyectos, se deberá entonces proceder a ejecutarlos. Este proceso de ejecución puede durar de 2 a 4 años, dependiendo del tipo de intervención requerida y decidida por las autoridades del Ministerio de Salud. Los diferentes aspectos de la recuperación hospitalaria se muestran en el Cuadro 3, distribuidos en el tiempo y con los costos aproximados. Puede observarse que se estima que la recuperación y rejuvenecimiento de la red puede tomar 4 años y con un costo que supera los 150 millones de dólares. Esto, claro está, basado en el supuesto de que los proyectos de refuerzo, arquitectura y equipamiento se puedan realizar en seis meses. Todo tiempo utilizado en decidir qué hacer, repercutirá en el plan de avance de la recuperación de la red.

Cuadro 3. Programación y costo de la recuperación hospitalaria (Los costos utilizan la moneda en dólares de los Estados Unidos de América)

\begin{tabular}{|c|c|c|c|c|c|c|c|c|}
\hline \multirow[t]{2}{*}{ Acciones } & \multicolumn{8}{|c|}{ MES } \\
\hline & 1 & 2 & 3 & 4 & 5 & 6 & Del 7 al 13 & Del 14 al 48 \\
\hline $\begin{array}{l}\text { Estudios de } \\
\text { vulnerabilidad }\end{array}$ & 386667 & 386667 & 386667 & & & & & \\
\hline Estudios de redes & 60000 & 60000 & 60000 & & & & & \\
\hline $\begin{array}{l}\text { Proyectos de } \\
\text { arquitectura }\end{array}$ & & & 1400000 & 1400000 & 1400000 & & & \\
\hline $\begin{array}{l}\text { Proyectos de } \\
\text { equipamiento }\end{array}$ & & & & & 300000 & 600000 & & \\
\hline $\begin{array}{l}\text { Proyectos de } \\
\text { mantenimiento }\end{array}$ & & & & & 300000 & 300000 & & \\
\hline $\begin{array}{l}\text { Proyecto refuerzos } \\
\text { arquitectónicos, etc. }\end{array}$ & 420000 & 420000 & & & & & & \\
\hline Obras a corto plazo & 3946000 & & & & & & & \\
\hline Obras a mediano plaz & & 1769750 & 1769750 & 1769750 & 1769750 & 1769750 & 12388250 & \\
\hline Obras a largo plazo & & & & & & & 19600000 & 98000000 \\
\hline Monto del período & 4812667 & 2636417 & 3616417 & 3169750 & 3769750 & 2369750 & 31988250 & 98000000 \\
\hline Monto acumulado & 4812667 & 7449083 & 11065500 & 14235250 & 18005000 & 20374750 & 52363000 & 150363000 \\
\hline
\end{tabular}




\section{CONCLUSIONES}

1. La red hospitalaria salvadoreña quedó muy lesionada después de los sismos del 13 de enero y del 13 de febrero del año 2001, debido al gran daño no estructural en mucho de sus hospitales de primer nivel y de segundo nivel de atención.

2. El daño no estructural se debe a la gran flexibilidad de los sistemas estructurales y a la fragilidad de los sistemas no estructurales arquitectónicos. Los pórticos flexibles resultaron ser estructuras inapropiadas para estructurar los edificios hospitalarios y quedó demostrado que las deformaciones relativas superiores al 4 por mil causaron el daño no estructural. Deformaciones relativas por debajo de 4 por mil no produjeron daño no estructural. Para estructuras flexibles, limitar las deformaciones a estos niveles es una tarea muy difícil ante sismos intensos.

3. La recuperación de la red no pudo darse de manera inmediata por aspectos técnicos, administrativos, financieros y sociales $\mathrm{y}$ debido al estado de vasta emergencia en que quedó la República Salvadoreña.

4. La recuperación del sistema hospitalario salvadoreño requerirá de una redefinición de los servicios que brinda cada hospital en función de la demanda. Se requerirá un rejuvenecimiento de la red así como la redefinición de algunos servicios que brinda.

5. Serán necesarios cuatro años, como mínimo, para reforzar los hospitales y rejuvenecer la red hospitalaria, y poner de nuevo el sistema hospitalario al servicio de la comunidad.

\section{COLABORADORES}

Los conceptos esbozados en el Plan de Recuperación Hospitalaria, fueron planteados por los arquitectos Micaela Baroni y Juan Pablo Quaglia, durante una consultoría realizada en El Salvador para tal efecto.

Los análisis de deformaciones del Hospital de Zacatecoluca fueron realizados por el Ing. Stefan Salazar Burger, como parte de su trabajo de graduación en la Universidad de Costa Rica, (ref. 4).

\section{BIBLIOGRAFÍA}

Colegio Federado de Ingenieros y Arquitectos de Costa Rica. (1986). Código Sísmico de Costa Rica. Editor Jorge Gutiérrez G. (Editor). Editorial Tecnológica, Cartago.

International Code Council. (2000). International Building Code. Virginia, USA.

Pauly, T., Priestley, M. Seismic design of reinforced concrete and masonry buildings.

Salazar, S. (2001). Deformaciones de tres edificios sometidos a los sismos del 2001 en El Salvador. Informe de Trabajo de Graduación, Facultad de Ingeniería, Universidad de Costa Rica, diciembre.

\section{SOBRE EL AUTOR}

\section{Miguel Francisco Cruz Azofeifa}

Profesor Catedrático de la Universidad de Costa Rica y Consultor Privado

San José, Costa Rica

Teléfono: (506) 2569294

Facsímil: (506) 2572574

Correo electrónico: mfcruz@racsa.co.cr 
